\title{
Interstitial Granulomatous Dermatitis: Another Clinical Variant
}

\author{
Aikaterini Patsatsi $^{a}$ Aikaterini Kyriakou $^{a}$ \\ Eva Triantafyllidou ${ }^{b}$ Dimitrios Sotiriadis ${ }^{a}$ \\ a 2nd Dermatology Department, Aristotle University School of Medicine, \\ Papageorgiou Hospital, and ${ }^{\mathrm{b}}$ Department of Rheumatology, Aristotle University \\ School of Medicine, AHEPA Hospital, Thessaloniki, Greece
}

\section{Key Words}

Interstitial granulomatous dermatitis · Adalimumab - Rheumatoid arthritis

\begin{abstract}
A 70-year-old female patient presented with an eruption consisting of symmetrically distributed erythematous papules around the umbilicus 1 month after the cessation of adalimumab for the treatment of rheumatoid arthritis. Biopsy of a papule showed an interstitial granulomatous infiltrate in the dermis, without deposition of mucin. The lesions cleared only after re-initiation of treatment 2 months later. Interstitial granulomatous dermatitis is thought to be a distinct histopathological pattern, either drug induced or associated with rheumatoid arthritis or autoimmune collagen diseases. In our case, there was a distinct clinical presentation of interstitial granulomatous dermatitis, composed of symmetrically distributed indurated papules around the umbilicus as well as a mild granulomatous reaction pattern.
\end{abstract}

\section{Introduction}

Interstitial granulomatous dermatitis (IGD) is thought to be a distinct histopathological pattern. It is characterized by the presence of histiocytes in the dermis between the collagen bundles arranged separately or in groups. This pattern may be either drug induced or associated with rheumatoid arthritis (RA) or autoimmune collagen diseases. Here, we present a case of IGD with distinct clinical features in a female patient with RA. 


\section{Case Report}

A 70-year-old woman with a 10-year history of RA, who was under treatment with adalimumab at a dose of $40 \mathrm{mg}$ subcutaneously every 2 weeks for the last 2 years, decided to stop her injections. One month later, she presented to our department with an eruption consisting of symmetrically distributed erythematous papules around the umbilicus (fig. 1).

Biopsy of a papule showed a normal epidermis and a perivascular and diffuse infiltration of the dermis by lymphocytes, histiocytes and a few giant cells, without deposition of mucin (fig. 2 , fig. 3 , fig. 4). Chest X-ray examination, tuberculin skin (PPD) test as well as QuantiFERON-TB test were all negative. ACE levels were within normal limits.

The lesions showed no improvement after application of topical steroids and cleared only after re-initiation of adalimumab treatment 2 months later, at the same dose of $40 \mathrm{mg}$ subcutaneously every 2 weeks. This treatment decision was mainly made by the rheumatologists, as the patient had an exacerbation of RA.

\section{Discussion}

IGD most often presents either as an interstitial granulomatous drug reaction (IGDR) or as IGD with arthritis (IGDwA).

IGDR clinically presents as asymptomatic, annular, erythematous to violaceous plaques with a predilection for the intertriginous areas, medial thighs and inner aspects of the arms [1]. Causative medications include calcium channel blockers, ACE inhibitors, lipid-lowering agents, antihistamines, diuretics, anticonvulsants, ganciclovir, antidepressants, interleukin-1 antagonists, trastuzumab, thalidomide and anti-TNF agents [2-4].

Anti-TNF agents have been implicated in the pathogenesis of IGDRs with histological variations. Apart from the interstitial lymphohistiocytic infiltrate, focal vacuolar degeneration of the basement membrane, necrotic keratinocytes, interstitial eosinophils and mucin may be present [4]. Adalimumab has been reported twice to cause IGDR $[4,5]$.

IGDwA is a rare disorder described mostly in female patients with RA in association with a relapse of the disease or as a drug-related reaction. It usually presents with linear, elongated, dermal bands without epidermal changes on the trunk and extremities ('rope' sign) [6].

It is likely that IGDwA represents a reactive phenomenon. The origin of this disorder is still unclear. It has been stated to be caused by an antigen-antibody reaction with the formation of auto-antibodies, which may affect several organs (especially the skin and joints) [2]. The mechanism of IGDwA may also be related to the immune-modulating effects of biologic agents, which may alter the antigenicity of dermal collagen, elicit an immune response, or disturb the normal production of collagen in response to damage [6]. Moreover, the close association between the development of skin lesions after the initiation of anti-TNF therapy and the clearance or improvement of the skin lesions after discontinuation of TNF inhibitors suggests a role of the medication on the development of cutaneous lesions [4]. It is possible that anti-TNF agents enhance the likelihood of developing IGDwA in RA patients who have granulomatous diathesis, although the true incidence of IGDwA in RA patients with or without anti-TNF therapy 
is not known. On the other hand, TNF is involved in a number of processes which help maintain granuloma induction of adhesion molecules, including endothelial cell activation, as well as growth of new blood vessels and regulation of other inflammatory cytokines [7]. Anti-TNF antibodies reduce all the above-mentioned interrelated activities and thus may be efficacious in the treatment of IGD, while cessation of them may induce granuloma formation again.

In a similar case to ours, IGDwA developed after sudden discontinuation of anti-TNF therapy, and the restart of this medication led to the resolution of the skin lesions [7]. The fact that this disorder responds to immunomodulatory treatment is consistent with the hypothesis of an altered immunologic reactivity.

In our case, there was a distinct clinical presentation of IGD, composed of symmetrically distributed indurated papules around the umbilicus as well as a mild granulomatous reaction pattern. The whole reaction was probably induced by the cessation of adalimumab and the subsequent gradual exacerbation of RA, although traces of the medication might still have been in the patient's blood when the initial rash presented.

In conclusion, with the increasing use of biologics worldwide, awareness of the clinical spectrum of drug- or RA-associated granulomatous lesions is continuously enriched.

\section{Disclosure Statement}

We declare no conflict of interest. 


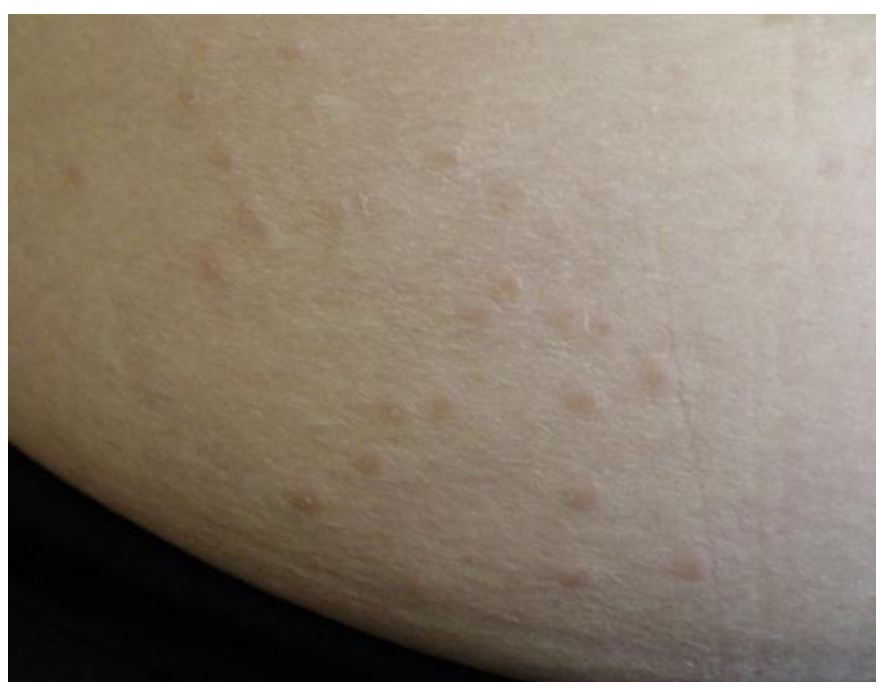

Fig. 1. Erythematous papules around the umbilicus.

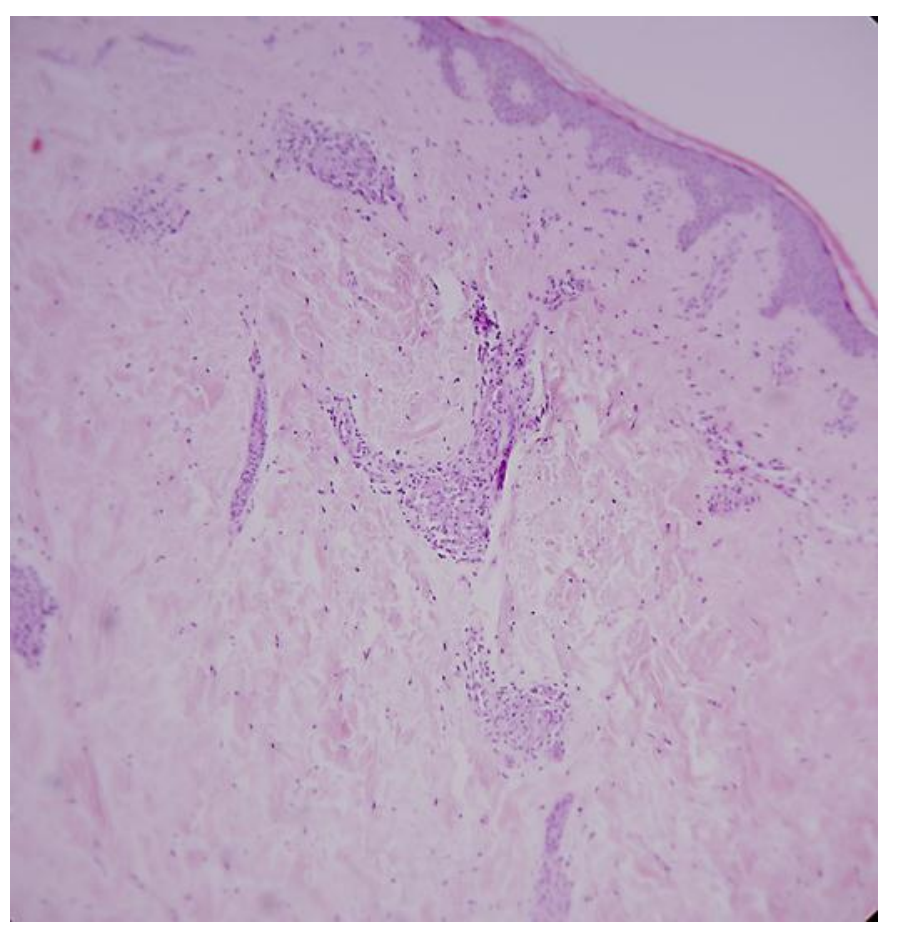

Fig. 2. Infiltration of the dermis by lymphocytes and histiocytes, without deposition of mucin $(\mathrm{HE} \times 10)$. 


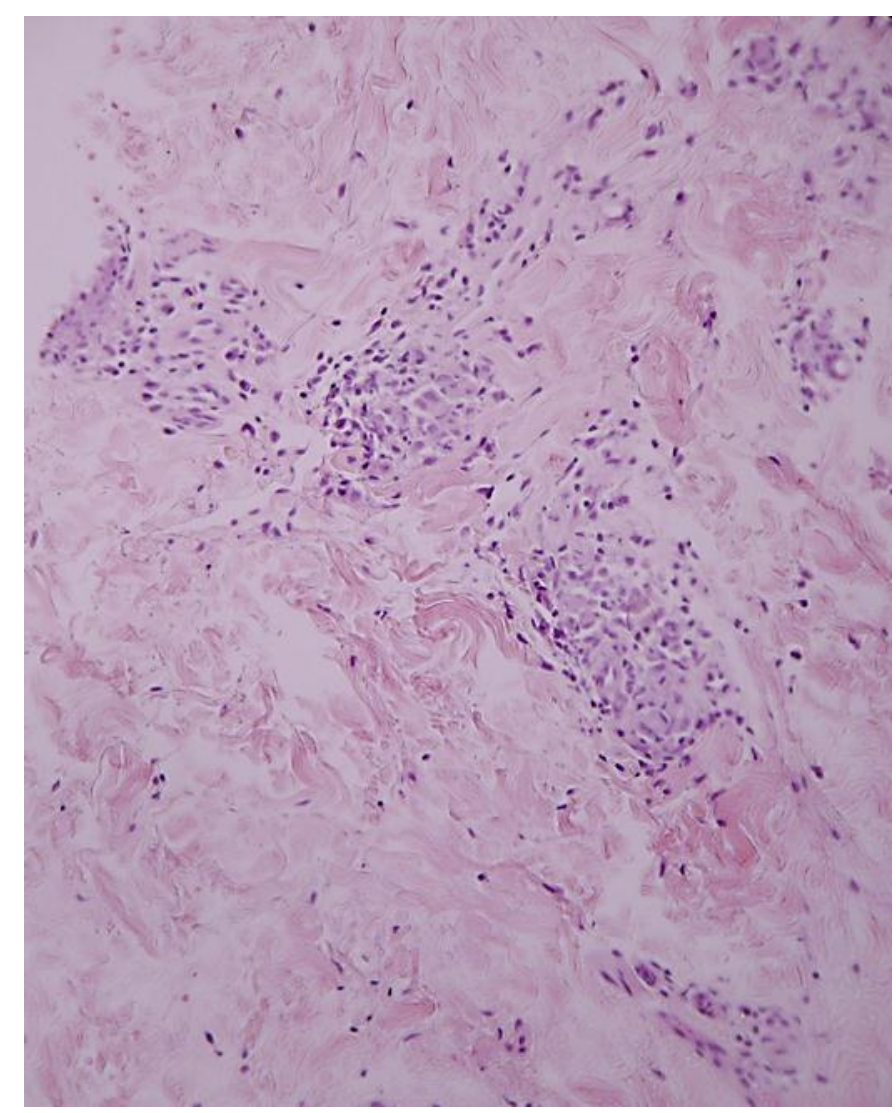

Fig. 3. Interstitial granulomatous infiltrate in the dermis $(\mathrm{HE} \times 20)$.

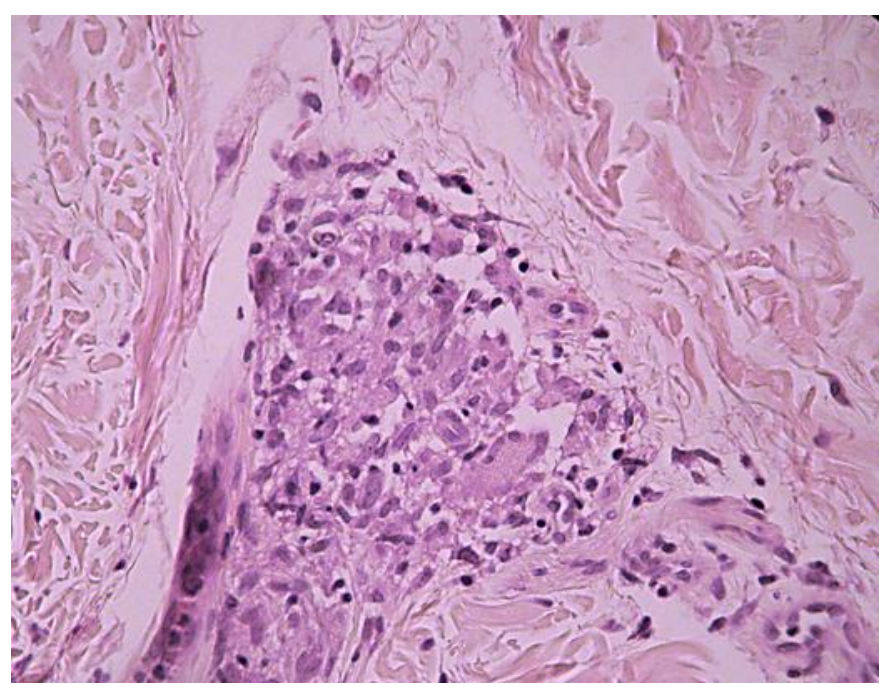

Fig. 4. Giant cell in the dermal infiltrate (HE $\times 40)$. 


\section{References}

1 Magro CM, Crowson AN, Schapiro BL: The interstitial granulomatous drug reaction: a distinctive clinical and pathological entity. J Cutan Pathol 1998;25:72-78.

2 Martin G, Canueto J, Santos-Briz A, Alonso G, Unamuno PD, Cruz JJ: Interstitial granulomatous dermatitis with arthritis associated with trastuzumab. J Eur Acad Dermatol Venereol 2010;24:493-494.

-3 Yazganoğlu KD, Tambay E, Mete 0, Ozkaya E: Interstitial granulomatous drug reaction due to thalidomide. J Eur Acad Dermatol Venereol 2009;23:490-493.

-4 Deng A, Harvey V, Sina B, Strobel D, Badros A, Junkins-Hopkins JM, Samuels A, Oghilikhan M, Gaspari A: Interstitial granulomatous dermatitis associated with the use of tumor necrosis factor alpha inhibitors. Arch Dermatol 2006;142:198-202.

5 Martorell-Calatayud A, Balmer N, Cardenas Cardona LF, Teague D: Interstitial granulomatous drug reaction to adalimumab. Am J Dermatopathol 2010;32:408-409.

-6 Regula CG, Hennessy J, Clarke LE, Adams DR, Ioffreda MD, Graber EM, Helm KF: Interstitial granulomatous drug reaction to anakinra. J Am Acad Dermatol 2008;59(2 suppl 1):S25-S27.

-7 Zoli A, Massi G, Pinnelli M, Lo Cuccio CD, Castri F, Ferraccioli G: Interstitial granulomatous dermatitis in rheumatoid arthritis responsive to etanercept. Clin Rheumatol 2010;29:99-101. 